\title{
ANL/MSD/CP-92654 CONF-970785-
}

\section{RBS ANALYSIS OF THE DIFFUSION OF NANO-SIZE SPHERES IN A POLYMER MATRIX*

\author{
D. H. Cole ${ }^{1,2}$, K. R. Shull ${ }^{2}$, L. E. Rehn' ${ }^{1}$, and P. M. Baldo ${ }^{1}$
}

${ }^{1}$ Materials Science Division

Argonne National Laboratory

9700 S. Cass Avenue, Argonne, IL 60439

2Department of Materials Science and Engineering

Northwestern University

2225 North Campus Drive

Evanston, IL 60205

August 1997

RECEIVED

AUG 261997

○ STI

\begin{abstract}
The submitted manuscript has been created
by the University of Chicago as Operator of

Argonne National Laboratory ("Argonne")

under Contract No. W-31-109-ENG-38 with

the U.S. Department of Energy. The U.S.

Government retains for itself, and others

acting on its behalf, a paid-up, non

exclusive, irrevocable worldwide license in

said article to reproduce, prepare derivative

works, distribute copies to the pubtic and

works, distribute copies to the pubtic, and

perform publicly and display publicly, by or
on behalf of the Government.
\end{abstract}

\section{DETREUTION OF THIS DOCUMENT IS UAHAMTED}

To be submitted to the Ion Beam Analysis-13 Conference, Lisbon, Portugal, July 27 - August 1, 1997.

\footnotetext{
*Work supported by the U.S. Department of Energy, Basic Energy Sciences-Materials Sciences, under contract \#W-31-109-ENG-38.
} 


\section{DISCLAIMER}

This report was prepared as an account of work sponsored by an agency of the United States Government. Neither the United States Government nor any agency thereof, nor any of their employees, make any warranty, express or implied, or assumes any legal liability or responsibility for the accuracy, completeness, or usefulness of any information, apparatus, product, or process disclosed, or represents that its use would not infringe privately owned rights. Reference herein to any specific commercial product, process, or service by trade name, trademark, manufacturer, or otherwise does not necessarily constitute or imply its endorsement, recommendation, or favoring by the United States Government or any agency thereof. The views and opinions of authors expressed herein do not necessarily state or reflect those of the United States Government or any agency thereof. 


\section{DISCLAIMER}

Portions of this document may be illegible electronic image products. Images are produced from the best available original document. 
RBS Analysis of the Diffusion of Nano-Size Spheres in a Polymer Matrix*

Douglas H. Cole ${ }^{1,2}$, Kenneth R. Shull ${ }^{2}$, L. E. Rehn ${ }^{1}$ and P. M. Baldo'

${ }^{1}$ Materials Science Division, Argonne National Laboratory, Argonne, IL 60439, USA ;

${ }^{2}$ Department of Materials Science and Engineering, Northwestern University, Evanston, IL 60205, USA

\section{Abstract}

Rutherford Backscattering Spectrometry (RBS) with 1.5-MeV He ions was employed to measure the depth distribution of gold particles sandwiched between two poly(tert-buytl acrylate) layers following various isothermal annealing treatments; temperatures were kept below $170{ }^{\circ} \mathrm{C}$ to prevent degradation of the polymer. Since PTBA also degrades when the ion beam impinges the sample, it was necessary to quantify the mass loss in order to obtain accurate diffusion coefficients. Although the total mass loss was substantial (40-60\%), a simple expansion of the energy-to-depth scale was sufficient to account for the beam-induced shrinkage. This result is attributed to the fact that the mass loss occurs very rapidly, and is uniformly distributed through the depth of the sample. The data reveal that the diffusion of the nanometer-size gold particles is substantially slower than is predicted by a simple physical model (Stokes-Einstein) for noninteracting particles. This slowdown is attributed to polymer bridging interactions, in which polymer strands bind to individual metal particles, thereby retarding their diffusion.

\section{Introduction}

Polymers see application in automobile tires and components, sporting equipment, and packaging for integrated circuits. This class of materials has some very appealing properties, most notably ease of processing. The low temperatures required to process typical polymers result in inexpensive manufacturing for many thousands of products. There are many applications where a polymer must come into contact with metal, forming an interface. To probe the physics of this situation, we assess the nature of the polymer-metal interactions using a model system.

The current article is concerned with relatively weak interactions in a system in which gold nanoparticles are dispersed in a poly (tert-butyl acrylate) (PTBA) matrix. Previous work ${ }^{1,} 2$ concerning dispersions of metal nanoparticles in polystyrene (PS) and poly(2-vinylpyridine) (PVP) matrices showed large variations in the strength of the polymer-metal interactions, even though PS and PVP differ only by the presence of a nitrogen atom in the phenyl ring of PVP. The work with 
PVP and PS examined the interactions between the polymer and particle via mobility measurements (diffusion measurements carried about above the polymer glass transition temperature, $T_{g}$ ), the contact angle of the polymer on a gold surface, as well as the morphological development via cross-sectional TEM.

By investigating the mobility of gold nanoparticles in a variety of polymer systems, it is hoped that information concerning the nature and strength of non-covalent polymer-metal interactions can be obtained. Further details concerning the diffusive behavior of gold particles in PTBA has been reported previously. ${ }^{3}$

\section{Experimental}

The PTBA system currently under investigation has the distinctive property that gold nanoparticles show detectable mobility in the polymer even at relatively high concentrations, i.e., those for which the particle to particle spacing immediately following deposition averages less than $10 \mathrm{~nm}$. The particles self-assemble as islands after thermal evaporation of gold onto a polymer thin film at an integrated thickness insufficient for a continuous metal film to form. Figure 1 shows a TEM micrograph of the particles as deposited on a $100 \mathrm{~nm}$ thick PTBA film. For the TEM samples, the polymer thin film is typically $100 \mathrm{~nm}$ in thickness, while for the diffusion experiments discussed later, the film thickness is typically much larger, varying from $300-500 \mathrm{~nm}$.

The sample, now with gold deposited on the surface, then has another layer of PTBA polymer placed on top of the existing layer, forming a sandwich. The two polymer layers can be of different chemical composition or molecular weight if desired. The samples studied here had polymer layers of identical composition, thickness, and molecular weight. The samples are shown schematically in Figure 2, along with the chemical structure of the PTBA.

The sandwich sample is broken in two pieces, reserving one half as a control. The other half is annealed in a vacuum oven above the glass transition temperature of the PTBA polymer, enabling the particles to diffuse through the sample. Due to the temperature dependence of the segmental motion in polymers, the required annealing time and temperature are related, so one has the option 
of tailoring the time required to perform the experiment by raising or lowering the annealing temperature. The only restriction on the annealing temperature is that one must avoid thermal degradation of the polymer. PTBA has been shown 4 to degrade to poly(acrylic acid) upon heating to temperatures in the vicinity of $180^{\circ} \mathrm{C}$, and results from our current work seem to indicate that partial decomposition can occur at temperatures as low as $145^{\circ} \mathrm{C}$.

Measurement of the gold particle diffusion coefficients provides a great deal of information about the nature of the polymer/metal composite system. Upon heating above $\mathrm{T}_{\mathrm{g}}$, the polymer behaves as a viscous liquid. The particles become mobile, and diffuse by Brownian motion. It should be noted that on the size scale of the particles, the force of gravity is negligible. The Stokes-Einstein equation,

$$
D=\frac{k_{B} T}{6 \pi \eta R}
$$

gives the rate at which a non-interacting spherical particle of radius $R$ will diffuse through a viscous Newtonian medium of bulk viscosity $\eta .^{5}$ The denominator of Equation 1 gives the resistance, $\zeta$, a fluid offers to a body moving through it. It can be viewed as the force, $F$, required to propel a particle at a velocity $v$, normalized by that velocity $(\zeta=F / v=6 \pi \eta R)$.

Oscillatory shear rheometry experiments performed on yield the following expression for the polymer viscosity:

$$
\log _{10} \eta=A+\frac{706}{T-T_{g}+59.31}
$$

where $\mathrm{T}$ is the temperature of interest $\left({ }^{\circ} \mathrm{C}\right), \mathrm{Tg}$ is the glass transition temperature of the PTBA in question, and $\mathrm{A}$ is a constant that varies with molecular weight. For PTBA of $7,000 \mathrm{~g} / \mathrm{mol}, \mathrm{A}=-$ 5.86 and $\mathrm{Tg}=42^{\circ} \mathrm{C}$. For PTBA of $77,000 \mathrm{~g} / \mathrm{mol}, \mathrm{A}=-6.371$ while $\mathrm{Tg}=49^{\circ} \mathrm{C}$ for all $\mathrm{M}$ greater than $21,000 \mathrm{~g} / \mathrm{mol}$. Equation [1] provides a framework on which to base our analysis of polymer- 
metal interactions. The Stokes-Einstein equation assumes that the particle and medium are noninteracting. Therefore, by comparing the diffusion of the particles predicted by the Stokes-Einstein equation to that determined experimentally, a measurement of the relative degree of interaction between polymer and particle can be obtained.

The diffusion profiles are obtained using Rutherford Backscattering Spectrometry (RBS) as the depth profiling technique. The polymer/metal samples are particularly well suited to this type of analysis, due to the very high sensitivity of backscattered helium nuclei to high atomic mass targets. Furthermore, relatively large particle diffusion distances $(\sim 1 \mu \mathrm{m})$ can be probed due to the comparatively small stopping cross section of the polymer matrix. The experimental geometry for all of our RBS scans has the beam, the sample normal, and the detector coplanar with the plane of sample tilt. The detector was $45^{\circ}$ from the beam path, and the sample is tilted $10^{\circ}$ away from the detector.

The energy deposited into the polymer chain by the ions is more than sufficient to break many of the bonds connecting the constituent atoms of the polymer. The results of this bond breaking depend upon the polymer involved. Polystyrene is known to cross-link in the ion beam, but the degradation of PTBA is more drastic. RBS spectra of the PTBA polymer do not show the oxygen peak that one would expect given the structure of the polymer $\left(\mathrm{C}_{7} \mathrm{H}_{12} \mathrm{O}_{2}\right)$. Figure 3 shows a profilometer trace across the area where the beam impinged on a $500 \mathrm{~nm}$ thick PTBA film. Extensive degradation (revealed by thinning in the irradiated portion of the film) is clearly evident.

The purpose of the depth profiling is to obtain particle motion data over time. If the overall thickness of the sample is changed during the ion beam irradiation, relating the RBS energy loss to a physical depth becomes problematic. To determine if the depth information was preserved intact, a sample was prepared in which four layers of polymer and four layers of gold were placed upon a silicon substrate. In Figure 4, the RBS spectra taken shortly after the irradiation began (Fig 4a), and after the sample ceased degrading (Fig 4b), are shown. The distance between the scale bars in Fig 4 show the relative spacing of the gold peaks before and after extensive irradiation. The lengths of the scale bars in Fig 4b, while smaller after the irradiation, remain directly proportional 
to those in Fig 4a. This result indicates that despite the beam damage, meaningful and quantitative diffusion distances can be obtained simply by rescaling the depth by calibrating the samples after irradiation to a sample of known thickness, measured by an alternate technique such as profilometry or ellipsometry.

Having established the validity of using RBS to measure depth distributions in a degradable matrix, it was then possible to extract diffusion coefficients from the sandwich samples of gold and PTBA. Figure 5 shows representative spectra from a sample of molecular weight $100,000 \mathrm{~g} / \mathrm{mol}$, which was annealed at $125^{\circ} \mathrm{C}$ for 1 hour. The silicon, carbon, and gold regions are marked, and the broadening of the gold peak in the annealed sample is clearly evident. Note the absence of an oxygen peak in both scans, which would be present at an energy of $0.626 \mathrm{MeV}$ given our experimental conditions.

The inset in Fig. 5 shows an expanded view of the gold peak. The peak shows symmetrical broadening about the peak center, and can be fit with the slab-error-function diffusion equation,

$$
\phi(x)=\frac{1}{2}\left\{\operatorname{erf}\left(\frac{x+z^{*}}{2 \sqrt{D t}}\right)+e r f\left(\frac{x-z^{*}}{2 \sqrt{D t}}\right)\right\},
$$

where $\phi$ is the volume fraction of gold particles, and $t$ is the annealing time. To ensure accuracy of the fit, the integrated intensity of the gold peak is used to determine the equivalent thickness, $z^{*}$. The annealing time is known, and the diffusion coefficient (D) is used as the fitting parameter. To further ensure accuracy, the assumed profile calculated by the diffusion equation is convoluted with a normalized Gaussian broadening function with a FWHM equal to the detector FWHM. The control sample is subjected to the same analysis, and a value of $D$ is obtained which corresponds to the initial depth distribution of particles. This "effective" diffusion coefficient (because no particle diffusion has taken place) is subtracted in quadrature from the diffusion coefficent of the annealed sample, to yield an accurate quantification of particle motion.

3. Results and Discussion 
It is instructive to compare the experimental values to those which theory would predict. If the Stokes-Einstein equation is obeyed, a gold particle with a radius of $1.5 \mathrm{~nm}$, diffusing through a polymer of $\mathrm{M}=7,000 \mathrm{~g} / \mathrm{mol}$ at $60^{\circ} \mathrm{C}$ would produce a diffusion coefficient $\mathrm{D}=1.1 \times 10^{-13} \mathrm{~cm}^{2} / \mathrm{s}$. The value obtained via the RBS experiment is $3 \times 10^{-15} \mathrm{~cm}^{2} / \mathrm{s}$. The experimental value is lower than the theoretical prediction by a factor of about 40. In Figure 6, the results of several experiments, using PTBA chains of varying molecular weight, are shown. One can see that the diffusion coefficient at a given temperature decreases with molecular weight. This is as expected, as higher molecular weight polymers will present more resistance to diffusion by virtue of increased viscosity. Again applying Equation 1 , this time to a polymer of $\mathrm{M}=100,000 \mathrm{~g} / \mathrm{mol}$ at $135^{\circ} \mathrm{C}(\eta=$ $261 \mathrm{~Pa} \cdot \mathrm{s})$, we obtain a predicted value for the particle diffusion coefficient $\mathrm{D}=7.6 \times 10^{-12} \mathrm{~cm}^{2} / \mathrm{s}$. This is also much different than the experimental value obtained via RBS, as the experimental diffusion coefficient is now over 600 times slower than the expected value, $\mathrm{D}=1.2 \times 10^{-14} \mathrm{~cm}^{2} / \mathrm{s}$. The particle's effect on the diffusion therefore is not simply due to the increased bulk viscosity of the matrix, since if it were, the slowdown would be independent of molecular weight. Note that the temperature dependence of the Stokes-Einstein theory matches well with the experimental data, despite the necessity to increase the effective particle radius in order to achieve a good fit to the data points. The lines present in Fig. 6 show the Stokes-Einstein equation thus adjusted to pass through the experimental data. The large adjustments in effective radius would seem to indicate that the PTBA/gold system is interacting quite strongly, given the large decreases in measured diffusion coefficient. The temperature dependence is given its shape primarily by the temperature dependence of the polymer viscosity, and suggests that the physical mechanisms for enabling particle diffusion have their roots in the same mechanisms that govern polymer motion.

Using the Stokes-Einstein equation to analyze the particle diffusion has the advantage that the terms in the equation are all physical, meaningful, and can be determined experimentally. The temperature can be measured, as can the viscosity of the medium and the particle radius. It also 
allows us to selectively reduce the effects of some of the variables in our system, by taking into account their effects on the terms of the equation.

The polymer viscosity will change with molecular weight, as a result of temporary entanglements that form between the individual polymer chains. ${ }^{6}$ The observed slowdown could thus be explained by the gold particles introducing additional entanglements in the system, effectively enhancing the viscosity. Another possibility for viscosity enhancement would be if the particles attached themselves to multiple chains through an adsorption-like grafting mechanism. The ease with which a polymer segment can detach itself from a gold particle (to be immediately replaced by another segment) will depend on its chemical affinity for the surface. Each monomer will contribute its share to this grafting, which would be very strong in the case of, for example, a thiol group (-SH), but much weaker for the bulky acrylates in our experiments. However, despite the relatively low strength of an individual interaction, several monomers will be attached to the gold particle simultaneously. The chain connectivity will provide an opportunity for a monomer, having detached due to thermal vibration, to immediately re-attach to the particle. Therefore, the removal of the entire chain segment requires the sequential detachment of several monomers simultaneously, a process which is kinetically unlikely. This chain segment is thus said to be kinetically grafted to the particle. By this reasoning, it is possible that several chains could bind themselves to one particle, the particle acting as a temporary crosslink for the chains. This would effectively increase the molecular weight of the combined system, thus increasing the apparent viscosity of the diffusing polymer-particle entity. These kinetically grafted chains affect the particle hydrodynamic radius as well. The kinetic grafting of polymer chains to the particle will increase the effective radius of the object diffusing through the polymer melt. The diffusion coefficient will decrease as a result of the larger particle size, an effect separate from the increase in apparent polymer viscosity.

With a sufficient degree of polymer/particle interaction, one would expect that the motion of the gold particles would eventually freeze out, as the particles get tied more and more tightly together. Extremely strong interactions would result in an effective network being formed, which should 
behave, at least qualitatively, like a crosslinked polymer network. That this phenomenon is not observed is evidence that if a network is formed, it must be transitory in nature. The dimensions of the polymer strands compared to the interparticle spacing would lead us to believe that an interacting network of bound strands must be present. The interparticle distance (which increases as the particles diffuse through the thickness of the film) is initially on the order of $10 \mathrm{~nm}$. The contour length, that is, the end-to-end distance between the chain ends when the chain is stretched to its longest conformation, ranges from roughly $13 \mathrm{~nm}$ in the case of $7,000 \mathrm{~g} / \mathrm{mol}$ polymer, to 195 $\mathrm{nm}$ for the $100,000 \mathrm{~g} / \mathrm{mol}$ polymer. However, the probability of finding a chain in this configuration is vanishingly small, since polymers in the melt state exist in a random walk configuration. The root-mean-square end-to-end distances give a better approximation of the dimensions of the polymer coil, and these range from $5 \mathrm{~nm}$ to $20 \mathrm{~nm}$ for the shortest and longest chains in our experiments. One can reasonably expect, then, that direct bridging of two particles by one chain can occur, and certainly, there will be interactions between chains that are each bound to a separate particle.

Diffusion does occur in the system, so any bridges which do form must be transitory. Interestingly, Ostubo has observed Newtonian behavior at low shear rates for a related system consisting of colloidal flocs bridged by polymer molecules. ${ }^{7} \mathrm{He}$ ascribed this phenomenon to the transitory nature of the polymer bridges. The situation involving colloidal flocs is analgous to the present case of nanoparticle diffusion by Brownian motion. The temperature dependence of the Stokes-Einstein equation is seen because the driving force is thermal, while the retarding forces arise from the segmental motion of the polymer chains, which also govern the temperature dependence of viscosity. These segmental motions must also be responsible for the forming and unforming of the polymer bridges.

In conclusion, we have performed experiments to probe the physics behind metal nanoparticulate diffusion in a liquid polymer matrix. RBS depth profiling was successfully applied to yield the diffusion information, despite extensive beam damage caused by the ion beam to the polymer. The experiments reveal that a large slowdown in diffusion occurs compared to 
theoretical predictions based on the Stokes-Einstein equation, but that the physics of the process can be explained by postulating the existence of transitory bridging interactions between individual metal particles and polymer strands.

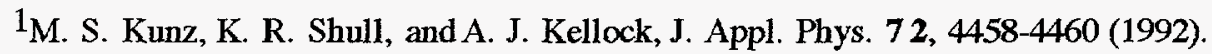

${ }^{2}$ M. S. Kunz, K. R. Shull, and A. J. Kellock, J. Coll. Int. Sci. 156, 240-249 (1993).

${ }^{3}$ D. H. Cole, K. R. Shull, L. E. Rehn, and P. Baldo, Physical Review Letters 7 8, 5006-5009 (1997).

${ }^{4}$ V. O. Cherkezyan and A. D. Litmanovich, European Polymer Journal 2 1, 623-626 (1991).

$5_{G}$. K. Batchelor, Journal of Fluid Mechanics 7 4, 1-29 (1976).

6P. Flory, Principles of Polymer Chemistry (Cornell University Press, Ithaca, NY, 1953).

${ }^{7}$ Y. Otsubo, Langmuir 8, 2336-2340 (1992). 
Figure Captions for IBA2-6.doc

Figure 1: TEM micrograph of Au particles deposited on a $100 \mathrm{~nm}$ poly (tert-butyl acrylate) thin film. The integrated thickness of the deposited gold is $0.4 \mathrm{~nm}$.

Figure 2: Chemical structure of poly(tert-butyl acrylate) and schematic of sample layup for Rutherford Backscattering experiments. The PTBA layers are each on the order of $0.4 \mu \mathrm{m}$ thick. The bulky tert-butyl group of the PTBA repeat unit is an excellent leaving group and is thought to degrade in the ion beam.

Figure 3: Profilometer trace across irradiated sample. The original film has lost perhaps $60 \%$ of the original thickness due to beam damage.

Figure 4: a) Initial RBS scan of layered PTBA sample cooled to liquid nitrogen temperatures to minimize initial degradation. The scale bars show the relative distance between gold peaks. b) An RBS spectrum of the same sample, taken after a long irradiation. The scale bars have the same ratio of lengths as in (a), which allows quantitative diffusion data to be extracted by a simple correction of the depth scale to account for the damage.

Figure 5: Sample RBS spectrum of a diffusional broadening experiment. The regions corresponding to carbon (polymer), silicon (substrate) and gold (diffusing particles) are labeled. The inset shows an expanded view of the gold peak. The broadening of the gold depth profile due to diffusion is clearly shown.

Figure 6: Gold particle diffusion coefficient vs. temperature for six polymer molecular weights. $M=7,000$ $\mathrm{g} / \mathrm{mol}(0), \mathrm{M}=21,000 \mathrm{~g} / \mathrm{mol}(\times), M=50,000 \mathrm{~g} / \mathrm{mol}(0), M=77,000 \mathrm{~g} / \mathrm{mol}(\Delta), M=85,000(\vec{t})$ ), and $\mathrm{M}=$ $100,000 \mathrm{~g} / \mathrm{mol}(+)$. The curve fits show the ability of the Stokes-Einstein temperature dependence to account for the variations in diffusion coefficient with temperature. At $\mathrm{T}>150^{\circ} \mathrm{C}$, thermal degradation of the polymer to poly (acrylic acid) may account for deviations from the model for the $\mathrm{M}=77,000 \mathrm{~g} / \mathrm{mol}$ and $\mathrm{M}=100,000 \mathrm{~g} / \mathrm{mol}$ polymers. 




Figure 1: TEM micrograph of Au particles deposited on PTBA thin film 


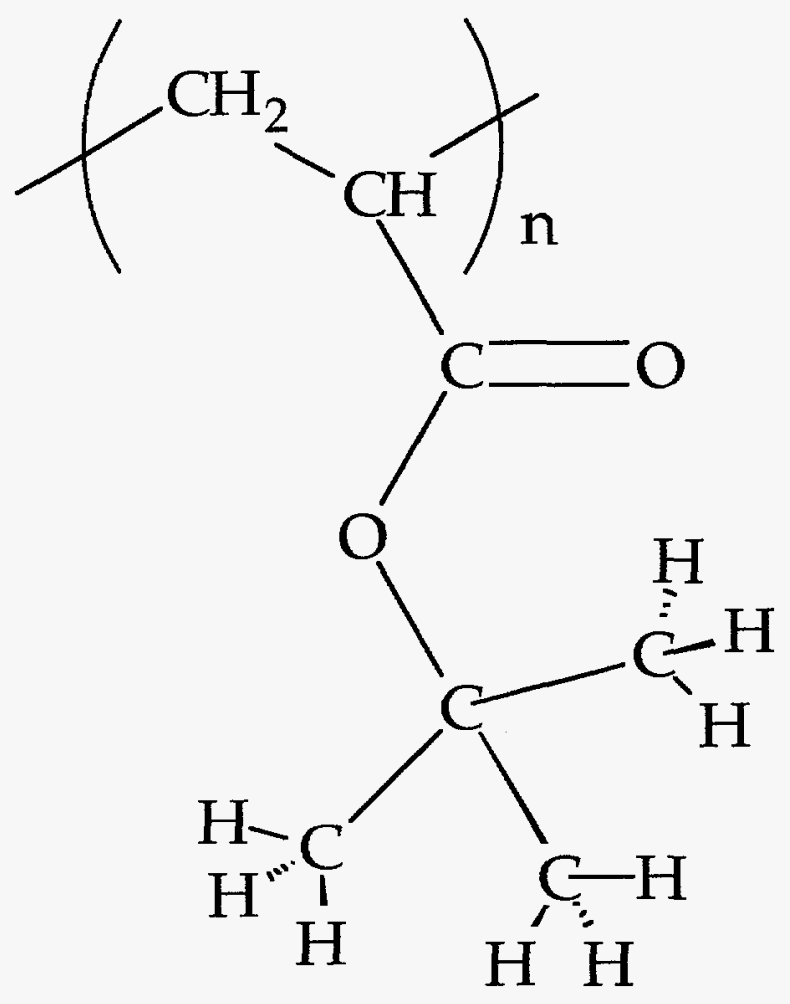

\section{PTBA}

Chemical Structure

PTBA Layer

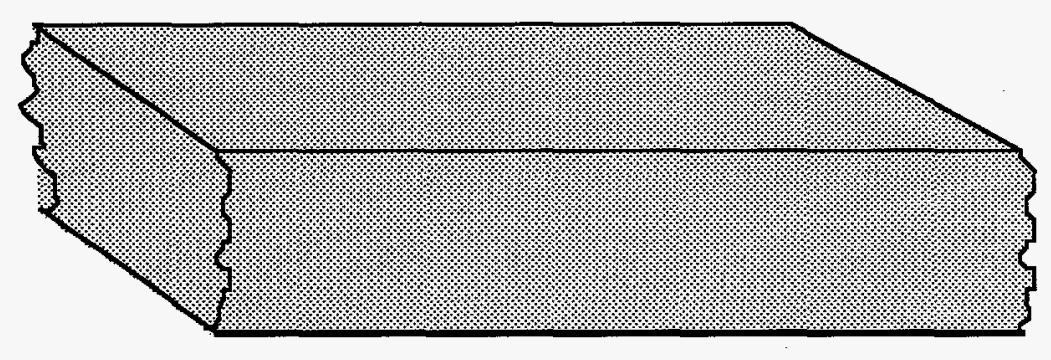

PTBA Layer

Silcon substrate

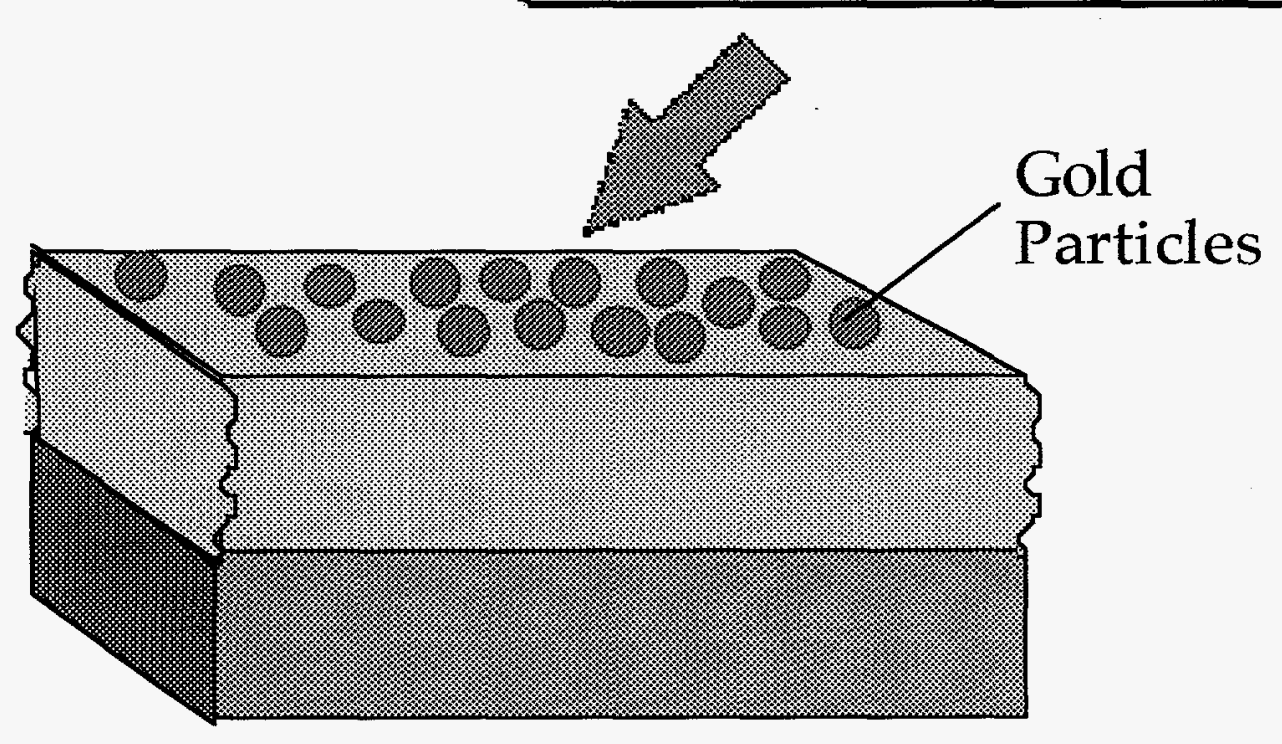




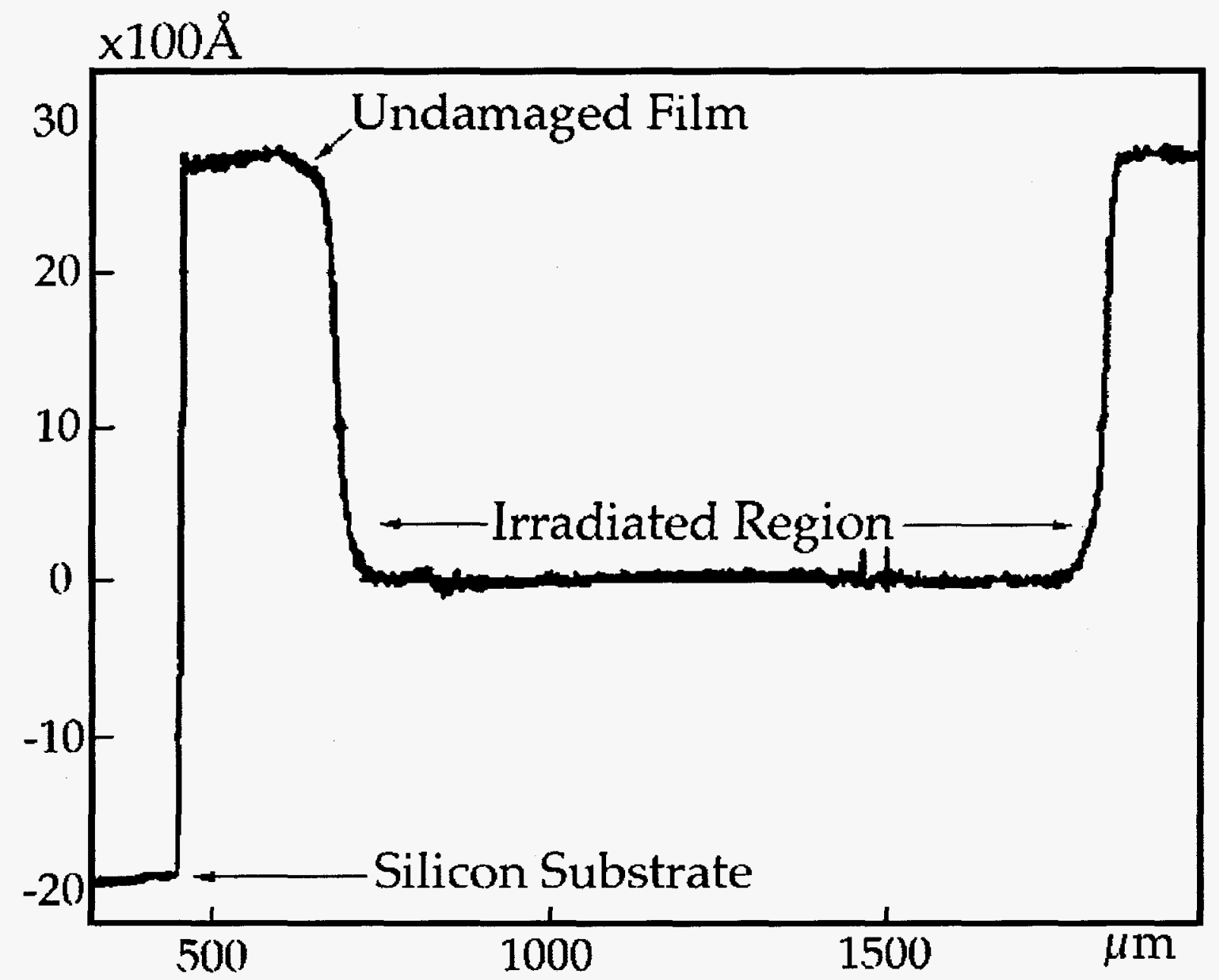




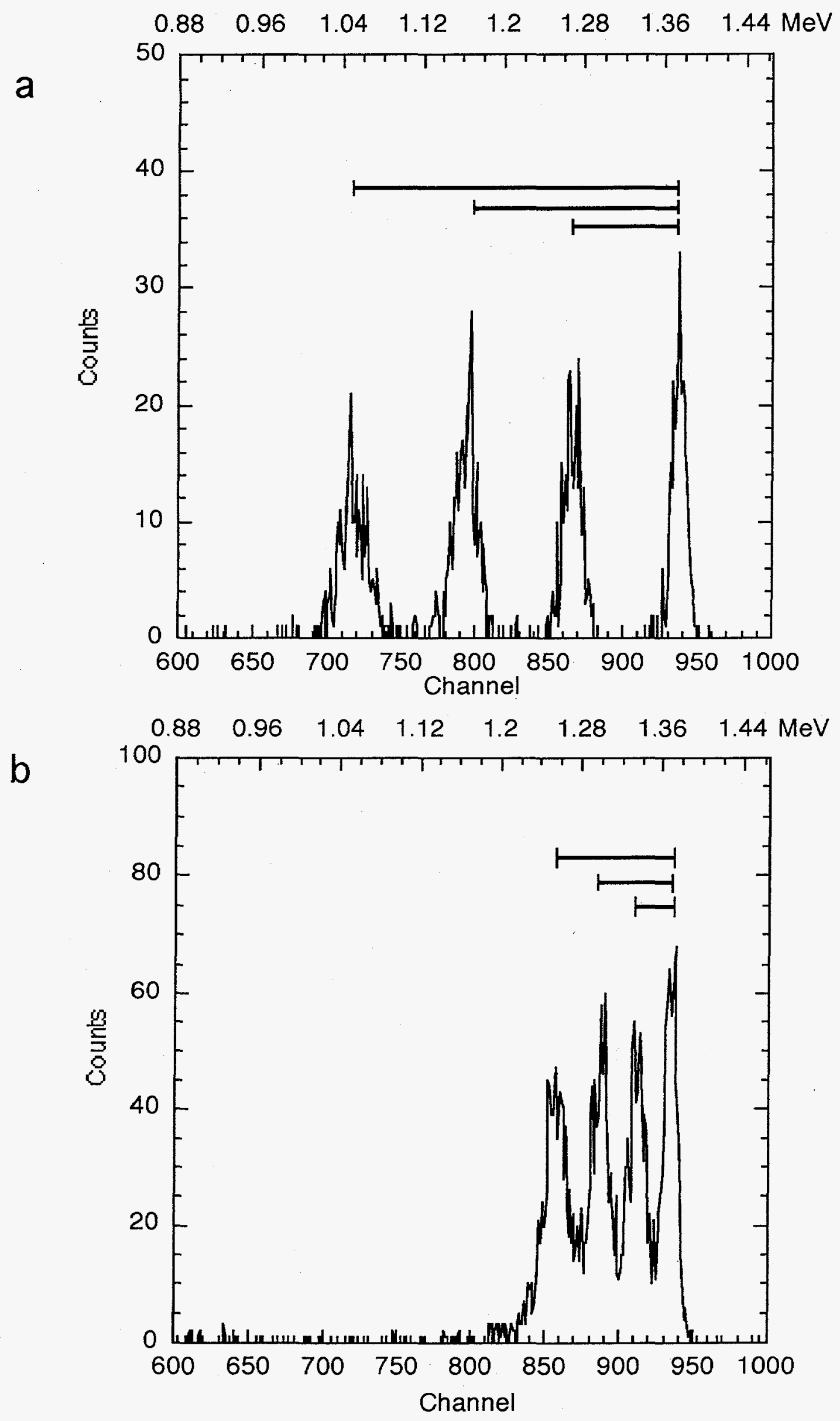




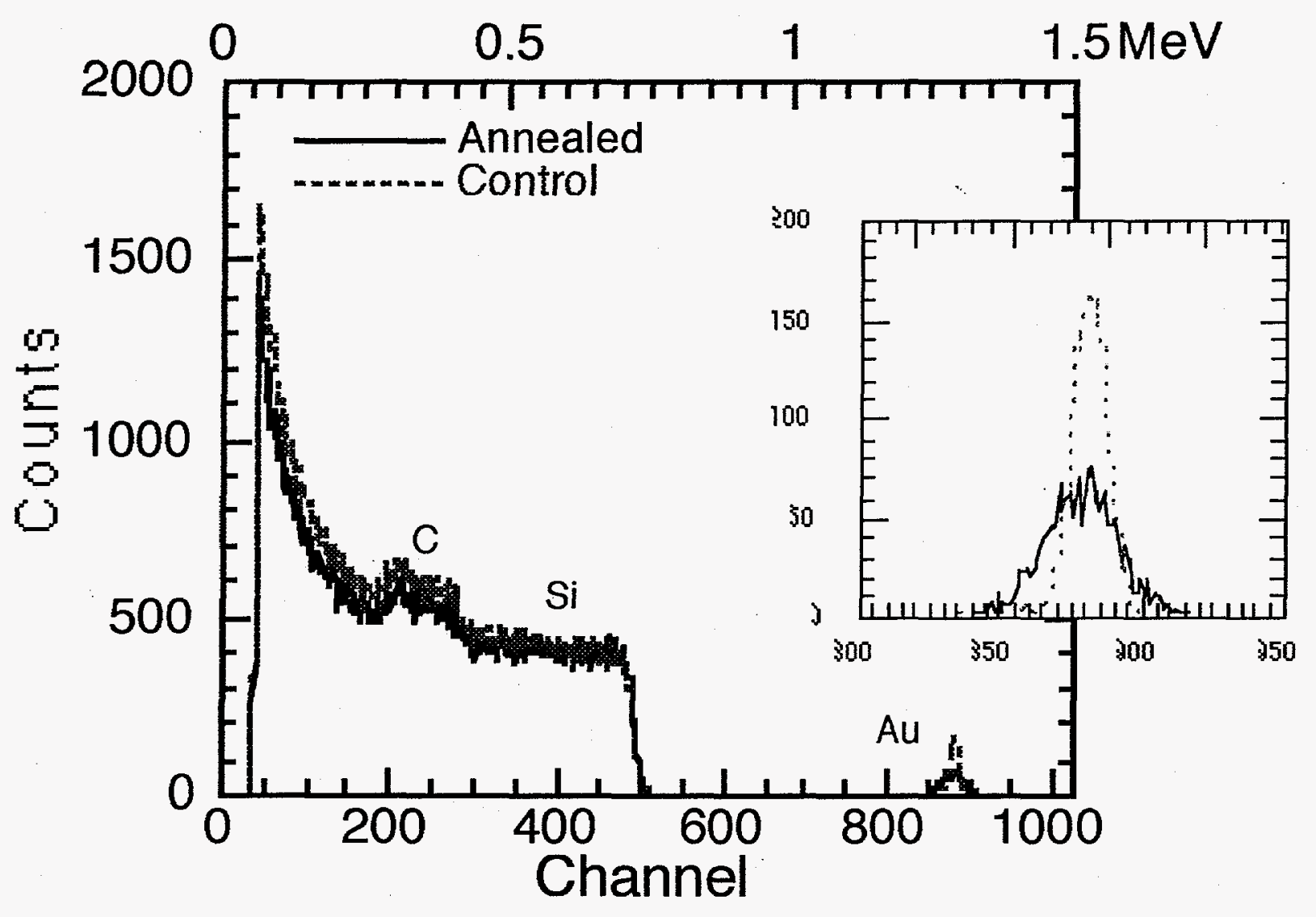




\section{Diffusion vs. Molecular Weight}

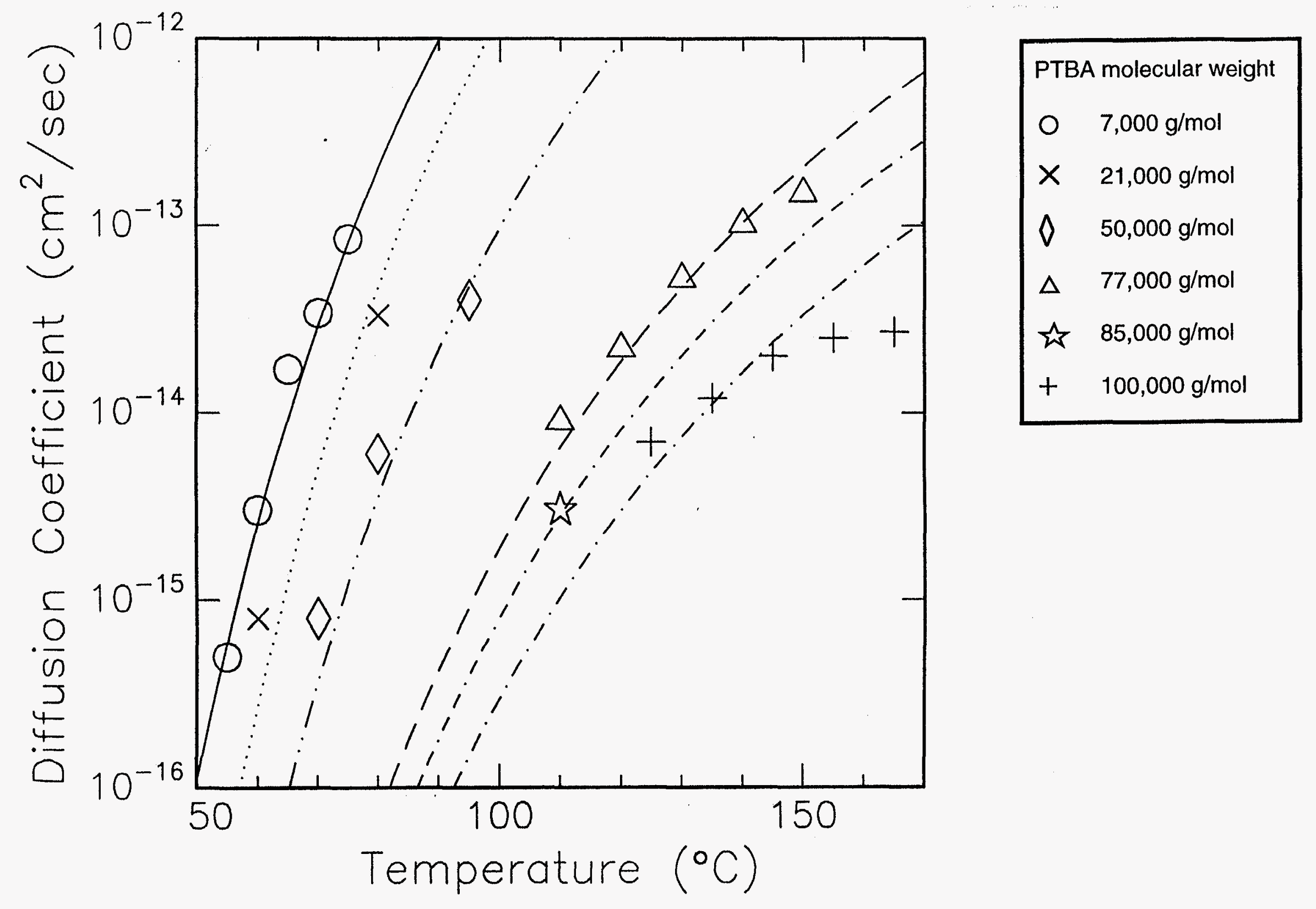

